\title{
MicroRNA-101 inhibits the metastasis of osteosarcoma cells by downregulation of EZH2 expression
}

\author{
KAILIANG ZHANG ${ }^{*}$, YINGLONG ZHANG ${ }^{*}$, KUN REN, GUANGYI ZHAO, KANG YAN and BAOAN MA \\ Department of Orthopedic Surgery, Tangdu Hospital, The Fourth Military Medical University, \\ Xi'an, Shaanxi 710038, P.R. China
}

Received May 6, 2014; Accepted August 11, 2014

DOI: $10.3892 /$ or.2014.3459

\begin{abstract}
MicroRNAs (miRNAs) are a class of small non-coding RNA molecules, which play regulatory roles at the post-transcriptional level by suppressing the translation of protein-coding genes or inducing mRNA cleavage. Dysregulated expression of miRNAs is involved in multiple types of cancers and plays important roles in regulating various biological processes including metastasis. miR-101 is downregulated in various types of cancer and functions as a suppressor of cell migration and invasion. Meanwhile, enhancer of zeste homolog $2(\mathrm{EZH} 2)$ is associated with the metastatic potential of several aggressive tumors. In the present study, we reported that ectopic overexpression of miR-101 downregulated the expression level of EZH2 and significantly inhibited migration and invasion of osteosarcoma cells. In addition, knockdown of EZH2 by siRNA showed the same effect of miR-101 on migration and invasion. To conclude, these results indicate that miR-101 may act as a tumor suppressor in osteosarcoma, as it has a suppressive role in cell migration and invasion by targeting EZH2.
\end{abstract}

\section{Introduction}

MicroRNAs (miRNAs) are small non-coding regulatory RNA molecules, 22 nucleotides in length, which suppress gene expression based on post-transcriptional suppression of target

Correspondence to: Professor Baoan Ma, Department of Orthopedics, Tangdu Hospital, The Fourth Military Medical University, Xi'an, Shaanxi 710038, P.R. China

E-mail: MA_B_A@126.com

${ }^{*}$ Contributed equally

Abbreviations: miRNAs, microRNAs; EZH2, enhancer of zeste homolog 2; miR-101, microRNA-101; FBS, fetal bovine serum; NC, negative control; MTT, 3-(4,5-dimethylthiazol-2-yl)-2,5-diphenyltetrazolium bromide; DMSO, dimethyl sulfoxide; OD, optical density; PRC2, polycomb repressive complex 2; H3K27, histone3 lysine27

Key words: microRNA-101, osteosarcoma, enhancer of zeste homolog 2, metastasis, migration, invasion
mRNAs by affecting mRNA translation or stability (1). It has been widely demonstrated that mRNAs are involved in the regulation of many cellular processes, such as cell differentiation, proliferation and apoptosis $(2,3)$. Recently, increasing evidence indicates that miRNAs are closely associated with cancer, and aberrant expression of miRNAs is often reported in cancers $(4,5)$. miRNAs can function as oncogenes or tumor suppressors, and play significant roles in tumorigenesis $(6,7)$. Those miRNAs, such as miR-21 and miR-155, whose expression levels tend to be upregulated in tumors, may be considered as oncogenes $(8,9)$. Meanwhile, some miRNAs, such as miR-143, miR-126 and miR-138, whose expression levels are usually downregulated in tumors, may be considered as tumor suppressors (10-12). These data indicate that miRNAs may be promising targets for tumor treatment.

Osteosarcoma is one of the most common primary malignant bone tumors and accounts for approximately $60 \%$ of all malignant bone tumors in childhood and adolescence (13). It is characterized by a high potential of lung metastasis (14), and for patients who develop lung metastasis, the survival rate is less than $30 \%$ (15). Therefore, to achieve a better prognosis, it is essential to identify the molecular mechanisms of osteosarcoma metastasis and to identify new therapeutic targets. Recent studies have confirmed the altered expression profile of miRNAs in osteosarcoma (16). Dysregulation of miRNAs, such as miR-183, miR-34a, miR-145 and miR-143, has been shown to be involved in the migration and invasion of osteosarcoma cells (17-20), which undoubtedly highlights the significance of miRNAs in osteosarcoma development and may reveal new insights into the mechanisms of metastasis.

MicroRNA-101 (miR-101) is a tumor-suppressor miRNA. It has been proven that miR-101 is downregulated in various types of cancer cells including lung cancer, gastric cancer, breast cancer and glioblastoma, and functions as a suppressor of cell migration and invasion (21-24). However, little is known concerning the functions of miR-101 in osteosarcoma. Therefore, it is of great importance to study the biological role and mechanism of miR-101 in osteosarcoma metastasis.

In the present study, we found that miR-101 expression in high-metastatic human osteosarcoma F5M2 cells was lower than that in low-metastatic human osteosarcoma F4 cells. Furthermore, ectopic overexpression of miR-101 inhibited migration and invasion of osteosarcoma cells by downregulating the expression level of EZH2, which was 
closely correlated with tumor metastatic potential (25-27). Overall, miR-101 may play a suppressive role in osteosarcoma metastasis through EZH2 downregulation, indicating that it may be a novel promising therapeutic target for osteosarcoma.

\section{Materials and methods}

Cell culture. The human osteosarcoma F5M2 and F4 sublines, which are respectively high-metastatic and low-metastatic cell lines and originate from the same human osteosarcoma SOSP-9607 cell line, were established and reserved by our laboratory (28). F5M2 and F4 cells were maintained in RPMI-1640 medium (HyClone, USA) supplemented with 10\% fetal bovine serum (FBS) (Sijiqing Co., China) and incubated at $37^{\circ} \mathrm{C}$ with $5 \% \mathrm{CO}_{2}$. The human osteosarcoma MG63 cell line was maintained under the same conditions except that MEM was used.

Transfection. Cells were transfected with miR-101 mimics and the NC mimics (negative control) (GenePharma Co., China) at a concentration of $100 \mathrm{nM}$, using Lipofectamine ${ }^{\mathrm{TM}} 2000$ reagent (Invitrogen Life Technologies, USA) according to the manufacturer's instructions. Efficiency of miR-101 transfection was detected by quantitative real-time PCR (qRT-PCR).

Quantitative real-time PCR analysis. Total RNA was isolated from cell lines with TRIzol reagent (Invitrogen Life Technologies) following the manufacturer's protocol. The miR-specific primers (Sangon Biotech Co., China) were designed for miR-101 qRT-PCR. A miR-specific primer (5'-ctcaactggtgtcgtggagtcggcaattcagttgagttcagttat-3') was used for reverse transcription. A pair of miR-specific primers (forward, 5'-acactccagctgggtacagtactgtgata-3' and reverse, 5'-tggtgtcgtggagtcg-3') were used on the ABI-Prism 7500 real-time PCR system (Applied Bioscience, USA) for amplification, compared with the normalization control U6 snRNA. A pair of primers (forward, 5'-gccagactgggaagaaatctg-3' and reverse, 5'-tgtgctggaaaatccaagtca-3') were designed for EZH2 qRT-PCR. To normalize the expression level of EZH2, GAPDH was used as an internal control. The relative expression level of mRNA was analyzed using the comparative $2^{-\Delta \Delta C t}$ method.

Migration and invasion assays. A total of $1 \times 10^{6}$ cells in low-serum media containing $1 \%$ FBS was added to the upper chamber of a Transwell system (24-well insert; pore size, $8 \mu \mathrm{m}$; Corning, USA). Six hundred microliters of complete media containing $20 \%$ FBS was added to the lower chamber. In the migration assay, cells that migrated to the outer surface of the membrane were fixed with $95 \%$ ethanol and stained with crystal violet after $24 \mathrm{~h}$ of incubation. In the invasion assay, the upper chamber was precoated with $50 \mu \mathrm{l}$ of Matrigel (1:8 dilution; BD Biosciences, USA). After $48 \mathrm{~h}$ incubation, the invaded cells were fixed and stained as described above. The cell numbers in 5 random fields were counted for each insert under a microscope (magnification, x200).

Wound healing assay. Adherent cell monolayers in 6-well plates were scratched with a $200-\mu 1$ pipette tip. The cells were then cultured in complete culture media at $37^{\circ} \mathrm{C}$ with $5 \% \mathrm{CO}_{2}$.
Wound healing ability was observed under microscopy after 0 , 24 and $48 \mathrm{~h}$ (magnification, x200).

MTT assay. Cell proliferation capacity was detected using the standard MTT [3-(4,5-dimethylthiazol-2-yl)-2,5-diphenyltetrazolium bromide] procedure, which was performed in 96-well plates. In brief, at $48 \mathrm{~h}$ after transfection, F5M2 cells were harvested and seeded at a density of 4,000 per well with $200 \mu \mathrm{l}$ complete culture medium. Each group contained 8 wells, and the wells without cells were used as blanks. Cell proliferation was documented every $24 \mathrm{~h}$ for 4 days; $20 \mu \mathrm{l}$ of $5 \mathrm{~g} / 1$ MTT (Sigma, USA) was added into each well. After $4 \mathrm{~h}$ of incubation at $37^{\circ} \mathrm{C}$, the media were removed and $150 \mu \mathrm{l}$ dimethyl sulfoxide (DMSO; Sigma) was added into each well. The optical density (OD) was evaluated according to the absorbance value detected at $490 \mathrm{~nm}$ with a reference of $630 \mathrm{~nm}$.

Western blot analysis. The western blot assay was carried out to detect the protein expression level of EZH2 following the standard method and this assay was performed with the EZH2 rabbit monoclonal antibody and the anti- $\beta$-actin mouse monoclonal antibody (Abcam, UK).

Statistical analysis. All experiments were performed at least in triplicate. The results are expressed as the mean values $\pm \mathrm{SD}$ and performed using SPSS 19.0 software. The Student's t-test was performed to determine the differences between groups, and $\mathrm{P}<0.05$ was considered to indicate a statistically significant difference.

\section{Results}

Expression of miR-101 in the F5M2 cells is lower than that in the F4 cells. To investigate the potential role of miR-101 in osteosarcoma metastasis, qRT-PCR analysis was carried out to compare miR-101 expression between the F5M2 and F4 cell lines. miR-101 expression in the F5M2 cells with high-metastatic potential was lower than that in the F4 cells with low-metastatic potential. The difference was statistically significant $(\mathrm{P}<0.05$, Fig. 1A).

Osteosarcoma cells transfected with miR-101 mimics show significant overexpression of miR-101. SOSP-9607 and MG63 cells were separately transfected with the miR-101 mimics at a concentration of $100 \mathrm{nM}$. qRT-PCR analysis was performed to detect the expression level of miR-101 at $48 \mathrm{~h}$ after transfection. The expression level of miR-101 was significantly upregulated in both the F5M2 and MG63 cells after treatment with the miR-101 mimics, compared with the F5M2 or MG63 cells treated with the NC mimics, respectively $(\mathrm{P}<0.01$, Fig. 1A and B). Meanwhile, there was no significant difference in miR-101 expression between the F5M2 cells treated with the NC mimics and untreated cells (Fig. 1A).

miR-101 significantly inhibits the migratory and invasive capacities of osteosarcoma cells. We used the Transwell assay to measure the migratory and invasive capacities of SOSP-9607 and MG63 cells. The results showed that osteosarcoma cells treated with the miR-101 mimics displayed significantly lower Transwell migration capacity, compared 

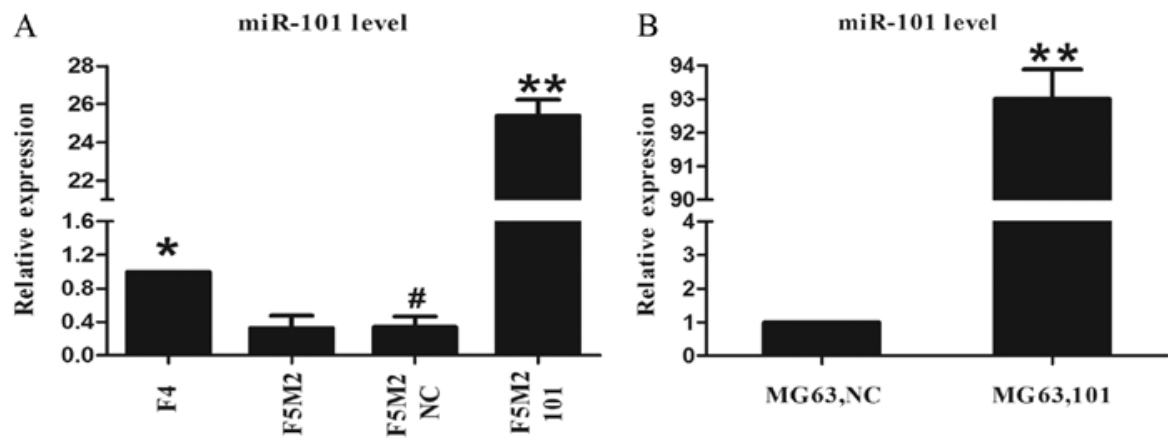

Figure 1. Efficiency of miR-101 transfection is verified by qRT-PCR. (A) miR-101 expression in the F5M2 cells was significantly lower than that in the F4 cells. Moreover, F5M2 cells transfected with the miR-101 mimics (F5M2 101) expressed a higher level of miR-101 when compared with the non-transfected cells (F5M2). Moreover, there was no difference between cells transfected with NC (F5M2 NC) and the non-transfected cells. (B) Compared with the cells transfected with NC (MG63,NC), MG63 cells following transfection with miR-101 mimics (MG63,101) expressed a higher level of miR-101. " $\mathrm{P}<0.05$, ${ }^{* *} \mathrm{P}<0.01$, compared with F5M2 or MG63 with NC; ${ }^{\text {P }}>0.05$, compared with F5M2.
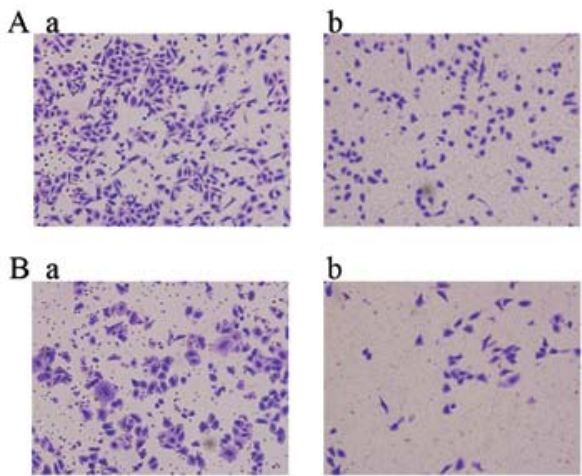

$\mathrm{C}$

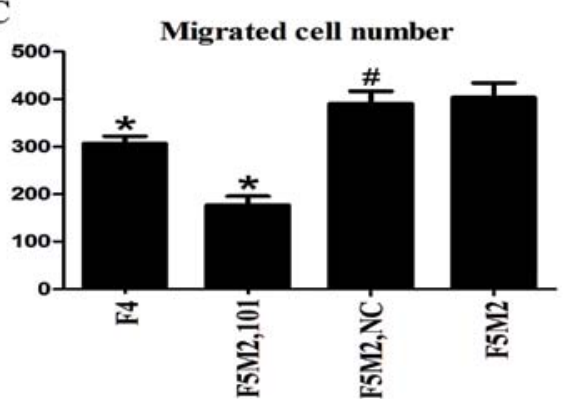

E a
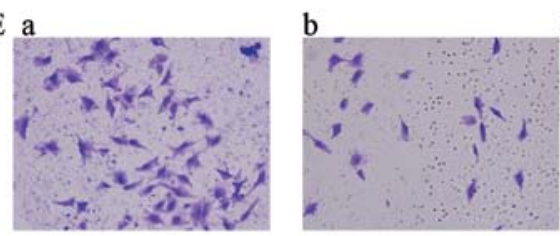

G

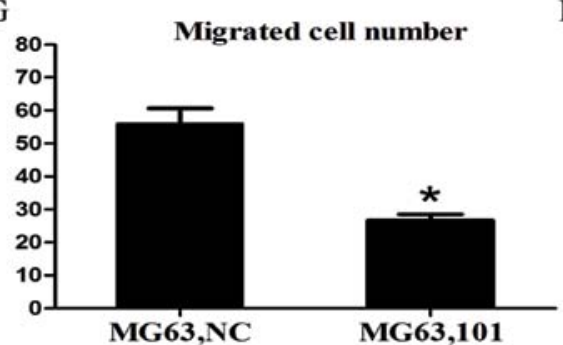

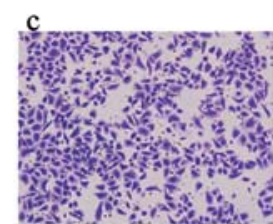
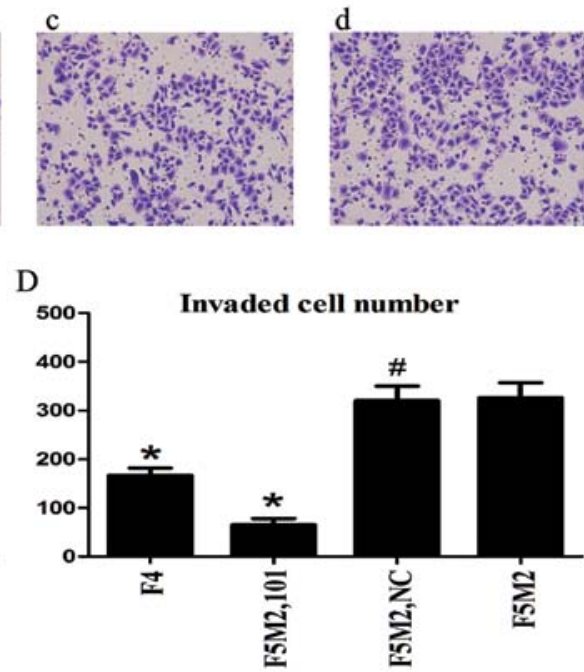

F a
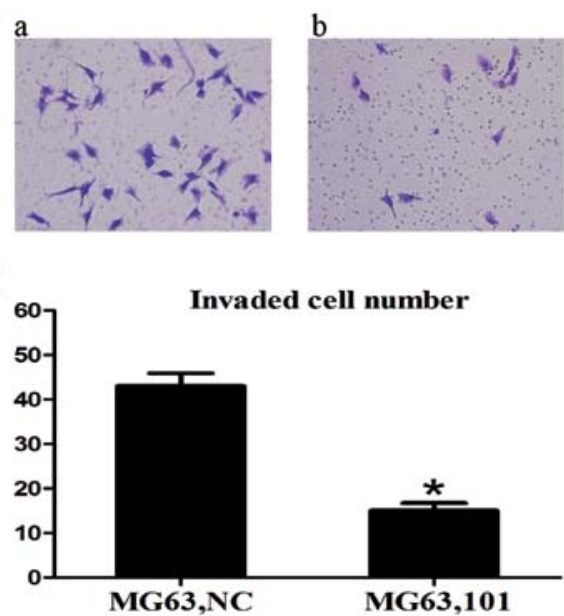

Figure 2. miR-101 inhibits migration and invasion of osteosarcoma cells in vitro. (A and B) Representative images of migrated and invaded SOSP-9607 cells under the microscope (magnification, x200). a, F4; b, F5M2 with miR-101; c, F5M2 with NC; d, F5M2. (C and D) F4 and F5M2 cells following transfection with miR-101 mimics (F5M2,101) showed less migratory and invasive ability than non-transfected F5M2 cells. (E and F) Representative images of migrated and invaded MG63 cells under a microscope (magnification, x200). a, MG63 with NC; b, MG63 with miR-101. (G and H) MG63 cells following transfection with miR-101 mimics (MG63,101) showed less migratory and invasive ability than cells transfected with NC (MG63,NC). "P<0.01, compared with F5M2 or MG63 with NC; ${ }^{\#} \mathrm{P}>0.05$, compared with F5M2. 

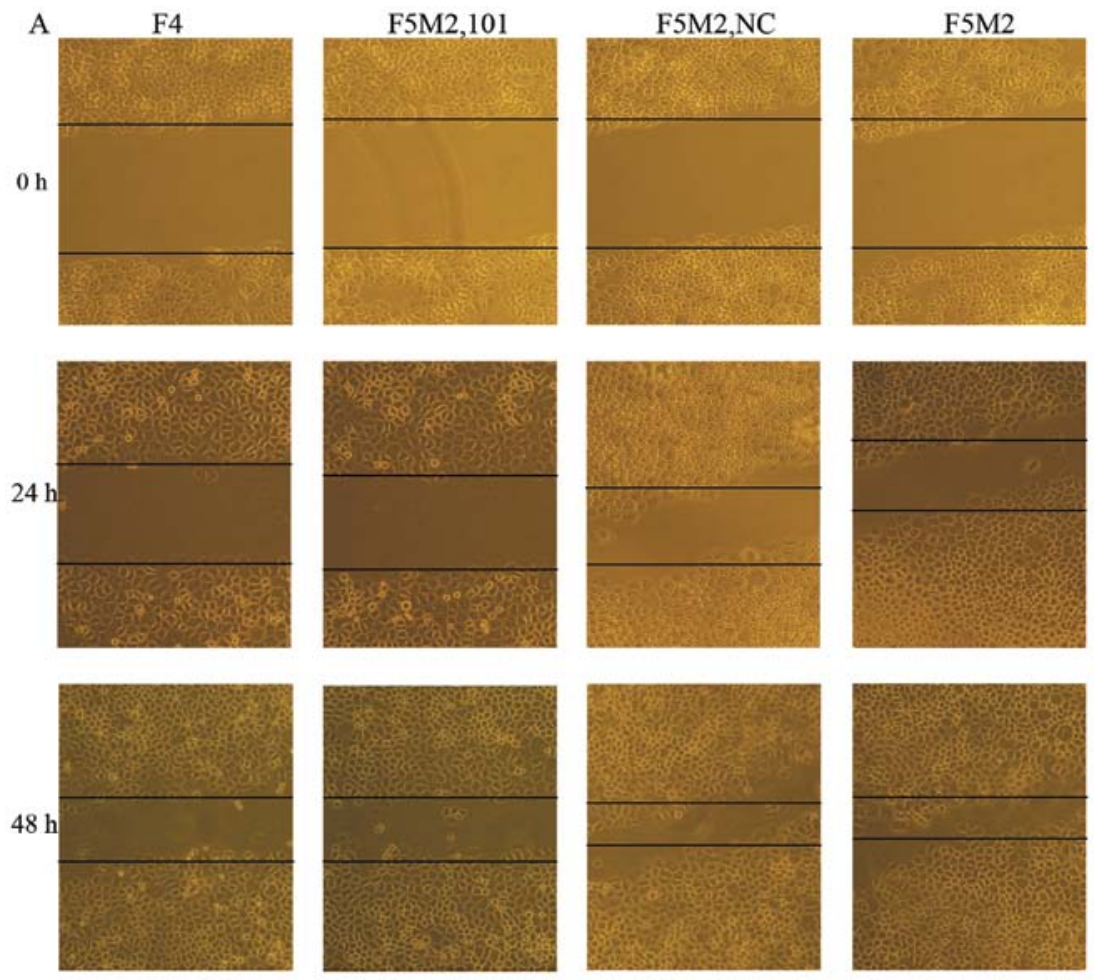

B

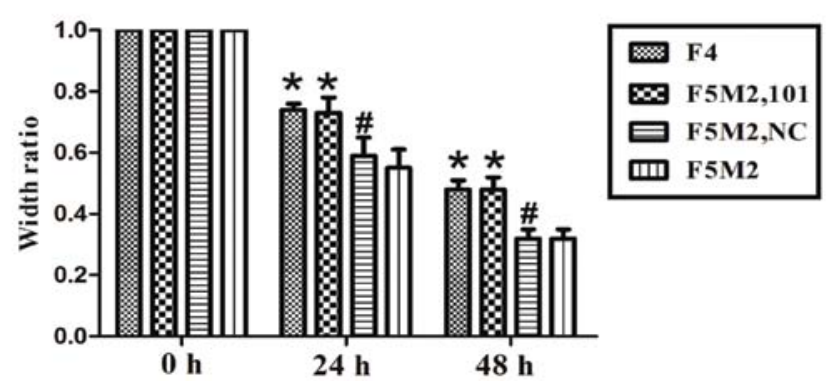

Figure 3. Wound healing assay. (A) Wound healing ability was observed under a microscope after 0,24 and $48 \mathrm{~h}$. (B) The data were analyzed and are summarized as width ratio $(0,24$ and $48 \mathrm{~h}$ width $/ 0 \mathrm{~h}$ width). The wound closure rates of the F5M2 cells transfected with the miR-101 mimics (F5M2,101) and the F4 cells were significantly lower than that of the F5M2 cells transfected with NC (F5M2,NC). Meantime, there was no statistically significant difference between the non-transfected F5M2 cells and F5M2 cells transfected with NC. ${ }^{*} \mathrm{P}<0.01,{ }^{\prime \prime} \mathrm{P}>0.05$, compared with F5M2.

with the cells untreated or treated with the $\mathrm{NC}$ mimics $(\mathrm{P}<0.01$, Fig. 2A and E). In the invasion assay, ectopic expression of miR-101 led to significantly decreased invasion of the osteosarcoma cells $(\mathrm{P}<0.01$, Fig. $2 \mathrm{~B}$ and $\mathrm{F})$. These results indicate a functional role for miR-101 in downregulating the migration and invasion of osteosarcoma cells.

miR-101 significantly inhibits the wound healing ability of osteosarcoma cells. We performed a wound healing assay and found that F5M2 cells closed the wounds faster than F4 cells $(\mathrm{P}<0.01$, Fig. 3$)$. Meanwhile, the migratory potential of the F5M2 cells treated with miR-101 mimics was significantly decreased when compared with that of the cells untreated or treated with the $\mathrm{NC}$ mimics $(\mathrm{P}<0.01$, Fig. 3).

miR-101 does not affect the proliferation of osteosarcoma cells. MTT assay was conducted to detect the potential role of miR-101 in the proliferation of osteosarcoma cells. The results indicated that there was no statistically significant difference between the F5M2 cells transfected with the miR-101 mimics

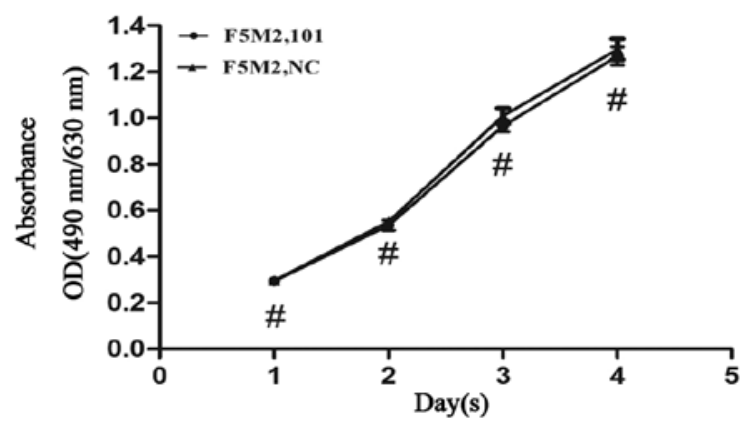

Figure 4. miR-101 has little effect on the proliferation of osteosarcoma cells in vitro. Every $24 \mathrm{~h}$, the MTT assay was performed on two groups of F5M2 cells [F5M2 cells transfected with miR-101 mimics (F5M2,101); F5M2 cells transfected with $\mathrm{NC}$ mimics (F5M2,NC)]. The cell number was analyzed as the value of the absorbance at $490 \mathrm{~nm}$ with a reference wavelength of $630 \mathrm{~nm}$. ${ }^{\#} \mathrm{P}>0.05$ is not considered statistically significant.

and the F5M2 cells transfected with the NC mimics. Therefore, miR-101 had little effect on the proliferation of osteosarcoma cells ( $\mathrm{P}>0.05$, Fig. 4). 


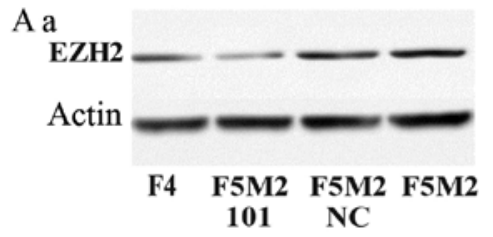

B a

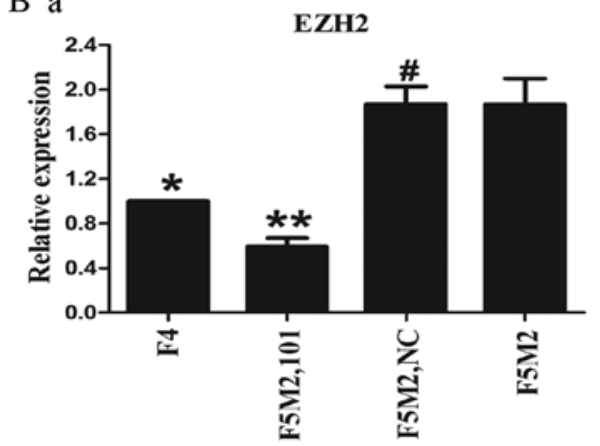

C a

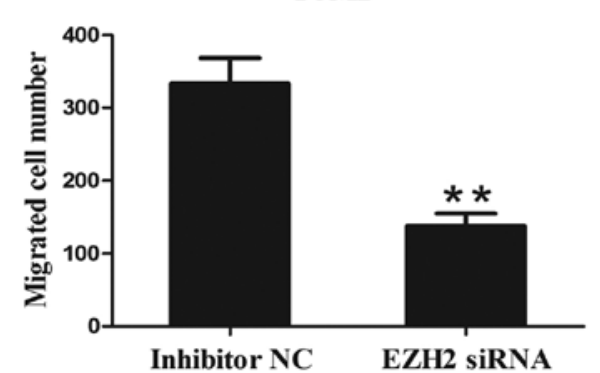

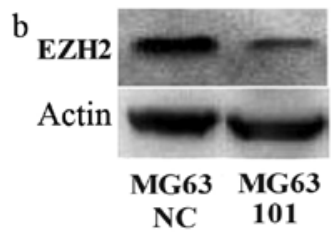

b EZH2

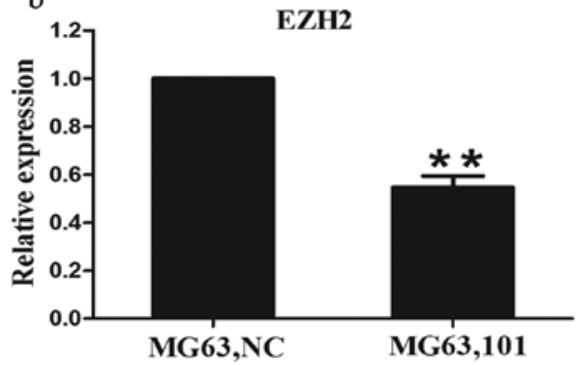

b

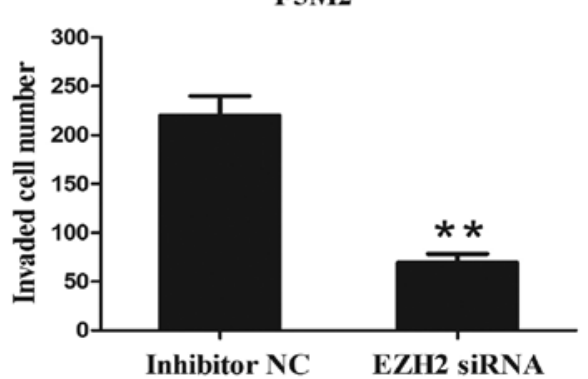

Figure 5. EZH2 is significantly downregulated by miR-101 at both the protein and mRNA levels, and regulates the migration and invasion of osteosarcoma cells. (A) Western blot analysis of EZH2 protein expression. (B) qRT-PCR analysis of EZH2 mRNA expression. In qRT-PCR analysis, GAPDH served as an internal control. (C) F5M2 cells were transfected with $100 \mathrm{nM}$ of EZH2 siRNA or inhibitor NC (GenePharma Co., China). Forty-eight hours later, the Transwell assay was employed. The results indicated that F5M2 cells in the EZH2 siRNA groups showed less migratory and invasive abilities than those in the inhibitor $\mathrm{NC}$ groups.* ${ }^{* *}<0.01$, is accepted as statistically significant. ${ }^{\#} \mathrm{P}>0.05$ is not considered to indicate a statistically significant difference.

EZH2 is significantly downregulated by miR-101 and regulates the migration and invasion of osteosarcoma cells. To analyze the expression of EZH2 in osteosarcoma, western blotting was performed on SOSP-9607 and MG63 cells. The results showed that the protein expression level of EZH2 in the F5M2 cells was higher than that in the F4 cells (Fig. 5A-a). Moreover, EZH2 expression was downregulated in the F5M2 cells treated with the miR-101 mimics compared to cells treated with the NC mimics or the untreated cells (Fig. 5A-a). MG63 cells treated with the miR-101 mimics also exhibited a lower expression level of EZH2 than cells treated with the NC mimics (Fig. 5A-b). In addition, qRT-PCR analysis displayed the same tendency at the mRNA expression level in both osteosarcoma cell lines (Fig. 5B). Then as determined in the Transwell assay, EZH2 siRNA significantly inhibited the migration and invasion of osteosarcoma F5M2 cells $(\mathrm{P}<0.01$, Fig. 5C). These results together reveal a negative correlation between miR-101 and EZH2 in osteosarcoma, and knockdown of EZH2 by siRNA inhibits the migration and invasion of osteosarcoma cells.

\section{Discussion}

In recent years, it has been widely demonstrated that miRNAs play profound roles in cancer pathogenesis and are implicated in numerous biological processes of a wide range of cancers, including metastasis. Aggressive tumor cell metastasis is a key step during cancer progression, and it contributes to secondary tumor formation at distant sites. Studies have reported that several miRNAs, such as miR-126, miR-335 and miR-145, are metastasis suppressors $(29,30)$. These data bring new insights into novel treatments of high-metastatic tumors including osteosarcoma, in which pulmonary metastasis is the leading cause of death (31).

Accumulating evidence indicates that miR-101 is significantly underexpressed and functions as a tumor suppressor in the migration and invasion of various types of cancers, such as lung cancer, gastric cancer, breast cancer and glioblastoma (19-22). However, little is known concerning the role of miR-101 in osteosarcoma. To investigate the potential role of miR-101 in osteosarcoma metastasis, we compared the expression levels of miR-101 in F5M2 and F4 cells, which are respectively high-metastatic and low-metastatic sublines of the human osteosarcoma cell line SOSP-9607 (26). Moreover, wound healing and Transwell assays were performed to assess the suppressive role of miR-101 in the migration and invasion of SOSP-9607 and MG63 cells. As expected, the F4 cells expressed a higher level of miR-101 than that in the F5M2 cells (Fig. 1A). Furthermore, miR-101 was significantly upregulated after transfection with the miR-101 mimics in 
both the SOSP-9607 and MG-63 cell lines (Fig. 1), and ectopic miR-101 significantly inhibited the migratory and invasive abilities of the osteosarcoma cells (Figs. 2 and 3). On the basis of these results, this study demonstrated the tumor-suppressive role of miR-101 in osteosarcoma metastasis for the first time, and miR-101 might be proven to be a promising therapeutic target in osteosarcoma.

It has been shown that miR-101 has multiple targets through which it has regulatory roles in the biological behaviors of various cancer cells. A previous study by Cho et al (21) showed that overexpression of miR-101 inhibited the invasive ability of lung cancer cells through suppression of EZH2. Furthermore, Wang et al (22) demonstrated that miR-101 expression in gastric tumor tissues and cells was higher than that in non-tumor gastric tissues and cells, and exogenous miR-101 inhibited the proliferation, migration and invasion of gastric cancer cells by downregulating the expression of EZH2, COX-2, Mcl-1 and FOS.

Predicting and identifying miR-101 target genes is important to further study the regulatory role of miR-101 in tumorigenesis and can offer a potential new therapeutic strategy for osteosarcoma. To investigate the suppressive mechanism of miR-101 in osteosarcoma metastasis, several prediction programs were used to seek the potential targets of miR-101. Markedly, the programs (including Target Scan, Pic Tar, Micro Inspector and Mir Target 2) predicted that EZH2 was one of the targets of miR-101.

Enhancer of zeste homlog 2 (EZH2) functions in a protein complex called the polycomb repressive complex 2 (PRC2), which is involved in the trimethylation of histone3 lysine27 (H3K27) and may lead to epigenetic silencing of target genes implicated in many cellular processes, including cell cycle regulation, cell differentiation and tumorigenesis (32). Enforced expression of EZH2 was initially found in prostate cancer, and EZH2 overexpression is closely correlated to aggressive and metastatic diseases (33). Subsequently, EZH2 was also found to be broadly overexpressed in a wide range of cancer types, such as breast cancer, bladder cancer, colon cancer, lymphomas and osteosarcoma (34-39). EZH2 has properties of oncogenes, as its overexpression has been correlated with the metastatic potential of several aggressive tumors (40-42). As for the effect of EZH2 in osteosarcoma, it has been reported that osteosarcoma patient biopsy specimens have higher expression levels of EZH2 than normal bone tissues, and overexpression of EZH2 was found in osteosarcoma cells by immunohistochemical assay. However, the aberrant expression of EZH2 is not correlated to osteosarcoma growth in vivo and in vitro (37). In addition, miR-26a has been reported to inhibit cell migration and invasion by targeting the EZH2 gene (43).

miR-101/EZH2 were reported to be deregulated in several types of cancer, including renal cancer (44), prostate cancer (45), gastric cancer (22), invasive squamous cell carcinoma (46), glioblastoma (24) and bladder transitional cell carcinoma (47), and were found to be significantly correlated with migration, invasion, and metastasis $(22,24,45)$.

In our study, we evaluated whether ectopic expression of miR-101 could inhibit the metastasis of osteosarcoma by suppressing the expression level of EZH2 in osteosarcoma cell lines. We revealed that substantial EZH2 suppression by miR-101 was detected at the mRNA and protein levels in SOSP-9607 and MG63 cells (Fig. 5A and B). In addition, knockdown of EZH2 by siRNA showed the same tendency as the effect of miR-101 on migration and invasion (Fig. 5C). We also revealed that overexpression of miR-101 had little role in cell proliferation (Fig. 4), which was consistent with the role of EZH2 in osteosarcoma cells (37). Taken together, we showed that EZH2 expression was inversely correlated with miR-101, and the expression level of EZH2 was positively correlated with the capacity of cellular migration and invasion. It is reasonable to conclude that miR-101 inhibits the migration and invasion of osteosarcoma cells by downregulating EZH2 expression.

In conclusion, this is the first in vitro study to provide new insights into the role of miR-101 in osteosarcoma. It shows that by downregulating the EZH2 expression level, miR-101 plays a suppressive role in cellular migration and invasion of osteosarcoma. Further studies in vivo are being conducted, and miR-101 may be a promising candidate prognostic biomarker and gene therapeutic agent for osteosarcoma treatment.

\section{Acknowledgements}

This study was financially supported by the National Natural Science Foundation of China (no. 81072194).

\section{References}

1. Bartel DP: MicroRNAs: genomics, biogenesis, mechanism, and function. Cell 116: 281-297, 2004.

2. Peters L and Meister G: Argonaute proteins: mediators of RNA silencing. Mol Cell 26: 611-623, 2007.

3. Carthew RW and Sontheimer EJ: Origins and mechanisms of miRNAs and siRNAs. Cell 136: 642-655, 2009.

4. Jansson MD and Lund AH: MicroRNA and cancer. Mol Oncol 6: 590-610, 2012.

5. Chen PS, Su JL and Hung MC: Dysregulation of microRNAs in cancer. J Biomed Sci 19: 90, 2012.

6. Esquela-Kerscher A and Slack FJ: Oncomirs - microRNAs with a role in cancer. Nat Rev Cancer 6: 259-269, 2006.

7. Hammond SM: MicroRNAs as tumor suppressors. Nat Genet 39: 582-583, 2007.

8. Qin X, Yan L, Zhao X, Li C and Fu Y: microRNA-21 overexpression contributes to cell proliferation by targeting PTEN in endometrioid endometrial cancer. Oncol Lett 4: 1290-1296, 2012.

9. Rather MI, Nagashri MN, Swamy SS, Gopinath KS and Kumar A: Oncogenic microRNA-155 downregulates tumor suppressor CDC73 and promotes oral squamous cell carcinoma cell proliferation: implications for cancer therapeutics. J Biol Chem 288: 608-618, 2013.

10. Liu L, Yu X, Guo X, et al: miR-143 is downregulated in cervical cancer and promotes apoptosis and inhibits tumor formation by targeting Bcl-2. Mol Med Rep 5: 753-760, 2012.

11. Frampton AE, Krell J, Jacob J, Stebbing J, Castellano L and Jiao LR: Loss of miR-126 is crucial to pancreatic cancer progression. Expert Rev Anticancer Ther 12: 881-884, 2012.

12. Liu X, Jiang L, Wang A, Yu J, Shi F and Zhou X: MicroRNA-138 suppresses invasion and promotes apoptosis in head and neck squamous cell carcinoma cell lines. Cancer Lett 286: 217-222, 2009.

13. Link MP: Osteosarcoma in adolescents and young adults: new developments and controversies. Commentary on the use of presurgical chemotherapy. Cancer Treat Res 62: 383-385, 1993.

14. Jaffe N: Osteosarcoma: review of the past, impact on the future. The American experience. Cancer Treat Res 152: 239-262, 2009.

15. Ferguson WS and Goorin AM: Current treatment of osteosarcoma. Cancer Invest 19: 292-315, 2001.

16. Novello C, Pazzaglia L, Cingolani C, et al: miRNA expression profile in human osteosarcoma: role of miR-1 and miR-133b in proliferation and cell cycle control. Int J Oncol 42: 667-675, 2013.

17. Zhao H, Guo M, Zhao G, et al: miR-183 inhibits the metastasis of osteosarcoma via downregulation of the expression of Ezrin in F5M2 cells. Int J Mol Med 30: 1013-1020, 2012. 
18. Yan K, Gao J, Yang T, et al: MicroRNA-34a inhibits the proliferation and metastasis of osteosarcoma cells both in vitro and in vivo. PLoS One 7: e33778, 2012.

19. Fan L, Wu Q, Xing X, Wei Y and Shao Z: MicroRNA-145 targets vascular endothelial growth factor and inhibits invasion and metastasis of osteosarcoma cells. Acta Biochim Biophys Sin 44: 407-414, 2012

20. Osaki M, Takeshita F, Sugimoto Y, et al: MicroRNA-143 regulates human osteosarcoma metastasis by regulating matrix metalloprotease-13 expression. Mol Ther 19: 1123-1130, 2011.

21. Cho HM, Jeon HS, Lee SY, et al: microRNA-101 inhibits lung cancer invasion through the regulation of enhancer of zeste homolog 2. Exp Ther Med 2: 963-967, 2011.

22. Wang HJ, Ruan HJ, He XJ, et al: MicroRNA-101 is downregulated in gastric cancer and involved in cell migration and invasion. Eur J Cancer 46: 2295-2303, 2010.

23. Wang R, Wang HB, Hao CJ, et al: MiR-101 is involved in human breast carcinogenesis by targeting Stathmin1. PLoS One 7 : e46173, 2012.

24. Smits M, Nilsson J, Mir SE, et al: miR-101 is downregulated in glioblastoma resulting in EZH2-induced proliferation, migration, and angiogenesis. Oncotarget 1: 710-720, 2010.

25. He LJ, Cai MY, Xu GL, et al: Prognostic significance of overexpression of EZH2 and $\mathrm{H} 3 \mathrm{k} 27 \mathrm{me} 3$ proteins in gastric cancer Asian Pac J Cancer Prev 13: 3173-3178, 2012

26. Ren G, Baritaki S, Marathe H, et al: Polycomb protein EZH2 regulates tumor invasion via the transcriptional repression of the metastasis suppressor RKIP in breast and prostate cancer. Cancer Res 72: 3091-3104, 2012.

27. Rao ZY, Cai MY, Yang GF, et al: EZH2 supports ovarian carcinoma cell invasion and/or metastasis via regulation of TGF-beta1 and is a predictor of outcome in ovarian carcinoma patients. Carcinogenesis 31: 1576-1583, 2010.

28. Chen X, Yang TT, Wang W, et al: Establishment and characterization of human osteosarcoma cell lines with differen pulmonary metastatic potentials. Cytotechnology 61: 37-44, 2009.

29. Negrini M and Calin GA: Breast cancer metastasis: a microRNA story. Breast Cancer Res 10: 203, 2008

30. Watahiki A, Wang Y, Morris J, et al: MicroRNAs associated with metastatic prostate cancer. PLoS One 6: e24950, 2011.

31. Wada T, Isu K, Takeda N, Usui M, Ishii S and Yamawaki S: A preliminary report of neoadjuvant chemotherapy NSH-7 study in osteosarcoma: preoperative salvage chemotherapy based on clinical tumor response and the use of granulocyte colonystimulating factor. Oncology 53: 221-227, 1996.

32. Sauvageau M and Sauvageau G: Polycomb group proteins: multifaceted regulators of somatic stem cells and cancer. Cell Stem Cell 7: 299-313, 2010.

33. Varambally S, Dhanasekaran SM, Zhou M, et al: The polycomb group protein EZH2 is involved in progression of prostate cancer. Nature 419: 624-629, 2002.
34. Hussein YR, Sood AK, Bandyopadhyay S, et al: Clinical and biological relevance of enhancer of zeste homolog 2 in triplenegative breast cancer. Hum Pathol 43: 1638-1644, 2012.

35. Changchien YC, Tatrai P, Papp G, et al: Poorly differentiated synovial sarcoma is associated with high expression of enhancer of zeste homologue 2 (EZH2). J Transl Med 10: 216, 2012.

36. Wang H, Albadine R, Magheli A, et al: Increased EZH2 protein expression is associated with invasive urothelial carcinoma of the bladder. Urol Oncol 30: 428-433, 2012.

37. Fluge $\varnothing$, Gravdal K, Carlsen E, et al: Expression of EZH2 and $\mathrm{Ki}-67$ in colorectal cancer and associations with treatment response and prognosis. Br J Cancer 101: 1282-1289, 2009.

38. Guo SQ and Zhang YZ: Overexpression of enhancer of zests homolog 2 in lymphoma. Chin Med J 125: 3735-3739, 2012.

39. Sasaki H, Setoguchi T, Matsunoshita Y, Gao H, Hirotsu M and Komiya S: The knock-down of overexpressed EZH2 and BMI-1 does not prevent osteosarcoma growth. Oncol Rep 23: 677-684, 2010.

40. Tong ZT, Cai MY, Wang XG, et al: EZH2 supports nasopharyngeal carcinoma cell aggressiveness by forming a co-repressor complex with HDAC1/HDAC2 and Snail to inhibit E-cadherin. Oncogene 31: 583-594, 2012.

41. Liu DC and Yang ZL: Overexpression of EZH2 and loss of expression of PTEN is associated with invasion, metastasis, and poor progression of gallbladder adenocarcinoma. Pathol Res Pract 207: 472-478, 2011.

42. Alford SH, Toy K, Merajver SD and Kleer CG: Increased risk for distant metastasis in patients with familial early-stage breast cancer and high EZH2 expression. Breast Cancer Res Treat 132: 429-437, 2012 .

43. Song QC, Shi ZB, Zhang YT, et al: Downregulation of microRNA-26a is associated with metastatic potential and the poor prognosis of osteosarcoma patients. Oncol Rep 31: 1263-1270, 2014.

44. Sakurai T, Bilim VN, Ugolkov AV, et al: The enhancer of zeste homolog $2(\mathrm{EZH} 2)$, a potential therapeutic target, is regulated by miR-101 in renal cancer cells. Biochem Biophys Res Commun 422: 607-614, 2012.

45. Varambally S, Cao Q, Mani RS, et al: Genomic loss of microRNA-101 leads to overexpression of histone methyltransferase EZH2 in cancer. Science 322: 1695-1699, 2008.

46. Banerjee R, Mani RS, Russo N, et al: The tumor suppressor gene rap1GAP is silenced by miR-101-mediated EZH2 overexpression in invasive squamous cell carcinoma. Oncogene 30: 4339-4349, 2011.

47. Friedman JM, Liang G, Liu CC, et al: The putative tumor suppressor microRNA-101 modulates the cancer epigenome by repressing the polycomb group protein EZH2. Cancer Res 69: 2623-2629, 2009 